\title{
Nanoparticle Labels for Co-localization and Correlative Imaging at High Spatial Resolution
}

\author{
R.M. Albrecht*, O. Olorundare**, J.A. Oliver***, and D.A. Meyer* \\ *Dept.of Animal Sciences, UW-Madison, 1675 Observatory Drive, Madison, WI 53706 \\ **Dept. of Pharmacology and Therapeutics, University of Ilorin, P.M.B. 1515, Ilorin, Nigeria \\ *** Dept. of Biological Sci., UW-Milwaukee, 3209 N. Maryland Ave., Milwaukee, WI 53211
}

Correlation of multiple factors is necessary to the understanding of biological function. Correlative relationships can be at many levels from organismal to submolecular and can involve both structure and function. Since biological activity takes place in the context of multi-molecular complexes, an understanding of cellular function requires not only identification of the various molecular species but an understanding of their physical relationships. Certain components can often be reasonably identified based on their unique structure. However, for many biological components, some type of labeling must be employed to reliably identify their presence and location. Simultaneous correlation of several components requires readily differentiated labels for each component. At the level of the light microscope, this can involve the use of chromatic, fluorescent, or certain opaque labels. At higher levels of spatial resolution requiring electron microscopy, labels must provide molecular or sub-molecular spatial resolution in order to identify and localize molecular species or even individual epitopes on a single molecule. [1]

For simultaneous multiple labeling in the light microscope, fluorescent dyes having different excitation and emission frequencies can be used. Small colloidal metal particles in the 1 to $10 \mathrm{~nm}$ size range coupled to active fragments of antibodies or active fragments of ligands provide specific probes for EM labeling at molecular and sub-molecular levels of resolution. Simultaneous multiple labeling is employed to accurately determine spatial relationships and co-localization patterns of different individual molecular species in multi-molecular complexes either internally or on cell surfaces. Particles of different sizes can also be employed, but results can be problematic because larger markers, when bound to an antigen, may block more neighboring binding sites than smaller particles, or they can mask smaller labels thereby making them undetectable. Also, larger particles may bind to more than one epitope per bead, thus making it difficult to compare or quantify signal densities. Additionally, spatial resolution is substantially reduced with larger marker sizes.

We have utilized two approaches to provide correlative labeling at high spatial resolution. One approach is to synthesize particles of different shapes [2]. Colloidal particles having spherical, various geometric, or other specific morphologies can be synthesized from stable metals that can be conjugated directly to antibodies, ligands or active fragments of antibodies or ligands. These particles can be synthesized in the 5 to $20 \mathrm{~nm}$ range and can be identified by shape in high resolution SEM or TEM imaging. The second approach also relies on EELS/ESI or high resolution STEM/EDX imaging to differentiate particles of different compositions [3,4]. Particles can be composed wholly of one species such as Au or can be multi-element, core-shell type particles such as Pt-Au or Pt-Pd. Colloidal nanoparticles of the same size as small as 2 to $3 \mathrm{~nm}$ in diameter, but having different elemental compositions including $\mathrm{Au}, \mathrm{Ag}, \mathrm{Pd}, \mathrm{Pt}, \mathrm{Ru}, \mathrm{Rh}, \mathrm{Fe}$, and others can be used as labels. Each type of particle is conjugated directly to a different primary antibody or antibody fragment. ESI imaging of conjugates on ultrathin sections is used to identify the different particle types. With this 
approach, labeling reactions can be compared directly, since labels are identified by their elemental composition. Also, the number of different reporters for multiple labeling immuno-EM can be substantially increased in comparison to conventional techniques. Images of particles can be overlaid on zero loss TEM images providing a direct correlation between multiple specific labels and biological structure [2]. The ESI approach requires very thin specimens to limit multiple interactions of the primary electrons with only one atom. EDX imaging on the other hand permits analysis and imaging of thick sections which could be used for stereo pairs or for tomographic images. Recent advances in instrument and beam technology have substantially increased resolution attainable both in terms of particle size and mass required for an unambiguous signal. In the current study, we utilized an aberration corrected FEI-Titan STEM coupled with a high resolution EDX detector [4]. The combination of beam stability, size, and energy allows individual particles in the 2 to $3 \mathrm{~nm}$ range adjacent to one another to be readily identified and differentiated (figure 1). Hence, the STEM/EDX approach provides the sensitivity to perform high resolution simultaneous labeling on thick sections and even in whole mount preparations.

\section{References:}

1. D.A. Meyer et al., Micros. and Microanal. 16 (2010) 33

2. R. Bleher et al., J Micros. 230 (2008) 388

3. R. M.Albrecht and D.A Meyer. Biological Low Voltage Scanning Electron Microscopy. Springer Science, NY, (2008) 171

4. D.A. Meyer et al., Micros. Microanal. 16 (Suppl 2) (2010) 992

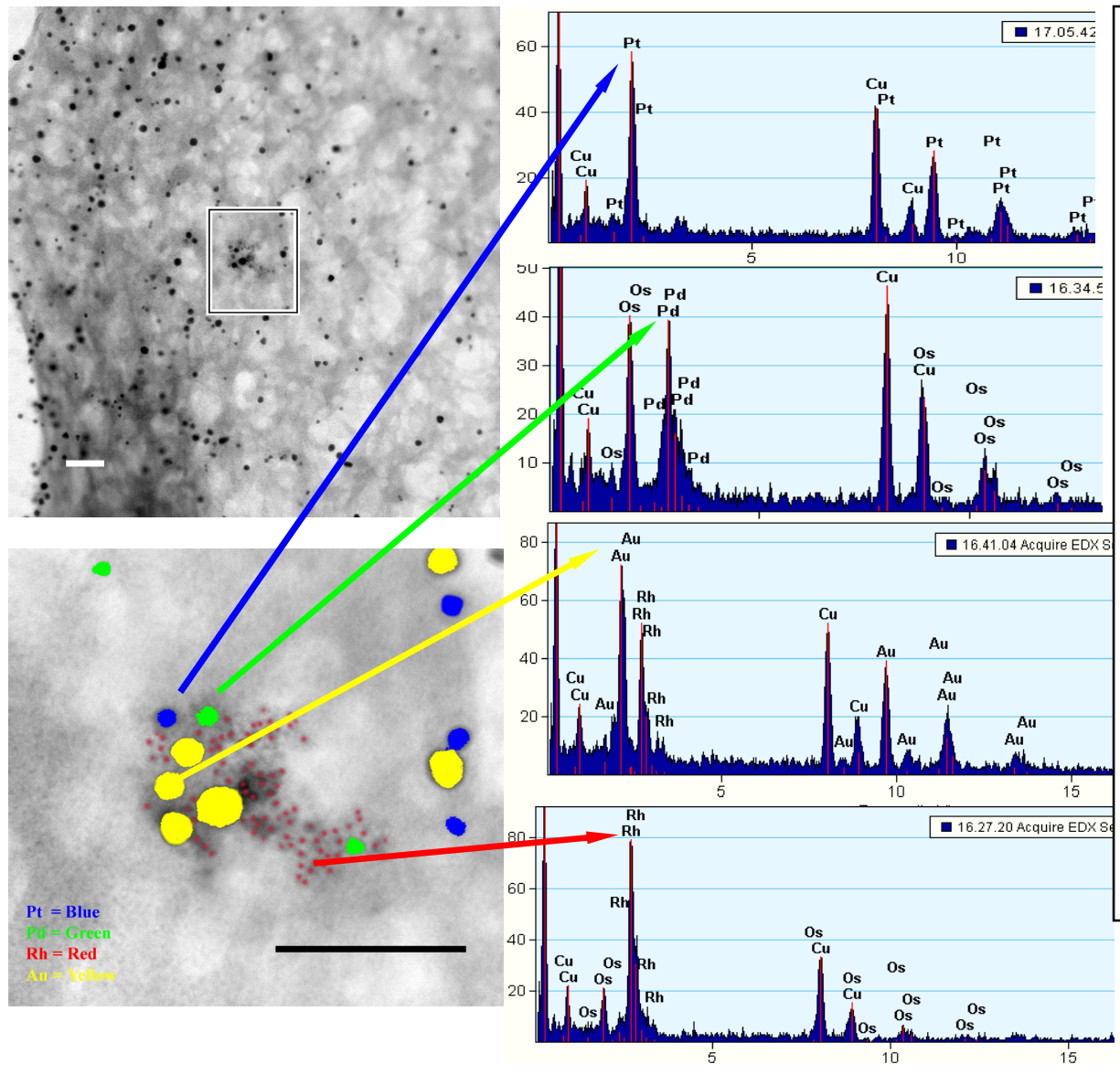

FIG. 1. Quadruple labeled platelet. Pt $6 \mathrm{~nm}$ conjugated to human prothrombin (blue); Pd $6 \mathrm{~nm}$ conjugated to anti alpha tubulin; (green); $\mathrm{Rh} 3 \mathrm{~nm}$ conjugated to fibrinogen (red); and $\mathrm{Au} 8 \mathrm{~nm}$ conjugated to human CD42b (GPIb) (yellow). Lower image corresponds to the area in the box in the upper image. Carbon peaks are from the support film, $\mathrm{Cu}$ peaks are from the grid, and Os peaks are from post-fixative, secondary staining. Scale bars are $50 \mathrm{~nm}$. 